\section{Reactions to various patterns of deceit in a mixed-motive game*}

\author{
ALAN A. BENTON† \\ University of Illinois, Chicago Circle, Chicago, Ill. 60680
}

Within the context of a two-choice mixed-motive experimental game, Ss were confronted by an opponent upon whom they were informationally dependent who either lied $25 \%$ or $50 \%$ for 32 trials and then was totally honest for the remaining (32) trials or who was totally honest for 32 trials and then lied $25 \%$ or $50 \%$. The more the other lied initially, the higher was the initial and subsequent rate of doubting of the Ss. Postgame data indicate that the Ss attributed greater control and responsibility to themselves when the other changed from dishonest to honest than when the converse occurred.

During the last 15 years, the most popular experimental situation used by the behavioral scientist for the study of interpersonal conflict resolution has been the experimental game. Most of these investigations have focused on the behavior of Ss in mixed-motive (or nonconstant-sum) games. One of the problems faced by a player in a game in which there are incentives to make both cooperative and competitive moves is how accurately to provide others with the information he possesses. Although effects of verbal communication in mixed-motive games have been studied in a variety of experimental game situations (Gruder, 1971; Loomis, 1959), there have been surprisingly few empirical investigations having any clear bearing on the reactions of individuals to deception and the variables which affect them (Benton, Gelber, Kelley, \& Liebling, 1969; Lindskold, Bonoma, Schlenker, \& Tedeschi, 1972; Tedeschi, Bonoma, \& Brown, 1971). The extensive program of research conducted by Tedeschi and his associates was concerned with the effects on cooperative responding in a prisoner's dilemma game of various levels of the credibility of announced intentions (in the form of promises and threats). Benton et al (1969) investigated the reactions of $\mathrm{Ss}$ to various degrees of deceit by a person upon whom they were informationally dependent.

The present study utilized the same experimental situation as that examined in the author's previous study: a mixed-motive relationship in which one of the players (A) has

* This research was supported by the Air Force Office of Scientific Research, Office of Aerospace Research, U.S. Air Force, under AFOSR Grant 1133-66 to Harold HI. Kelley. University of California, Los Angeles.

TRequests for reprints should be sent to the author, Department of Psychology, University of lllinois, Chicago Circle, Chicago, Ill. 60680 . cost-free knowledge of the successive states of a variable external to the players (a deck of cards) which indicates consequences for both of them. Person B can gain direct knowledge about the relevant state on any trial only at a cost which is levied against both members of the pair. As the trials proceed, B can either take A's word for the state (and thus permit the successive outcomes to be determined by A's assertions) or he can doubt A's statements (at their mutual expense) and insure that the distribution of rewards is uetermined by the cards. The purpose of this study was to investigate the reactions of individuals in the role of $B$ to persons in the role of $A$ who misrepresent the facts occasionally and who do so either during the early stages of the interaction or after they have been initially honest.

The Ss were exposed to one of four lie sequences. In two of the experimental conditions, a programmed $A$ accurately reported the information indicated by the cards during the first 32 trials of the interaction and then during the subsequent 32 trials lied either $25 \%$ or $50 \%$ of the time when the cards signified that $B$ was entitled to the reward (Conditions 0\%-25\% and $0 \%-50 \%$, respectively). On the one hand, one might expect that when $B$ discovers that $\mathrm{A}$ is lying, particularly in the $0 \%-50 \%$ condition, he may "overreact" to "catching" A. The disconfirmation of an expectation of honesty may result in a dramatic increase in B's rate of doubting. On the other hand, B may choose to avoid "inconsistent" information. Since present empirical evidence and theory lead to the conclusion that early honesty may either increase or decrease (or at least delay) subsequent doubting by B (Pruitt, 1969; Teger, 1970), no specific predictions were made with respect to the rate of doubting of the Ss in the $0 \%-25 \%$ and $0 \%-50 \%$ conditions. In the remaining two experimental conditions, the programmed A lied either $25 \%$ or $50 \%$ of the time during the first 32 trials and then was honest during the last 32 (Conditions 25\%-0\% and 50\%-0\%, respectively). It was expected that early lying would increase B's suspicion of A's assertions. Previous research (Benton et al, 1969) indicates that suspicious $\mathrm{Ss}$ are more likely to doubt A's communications than are trusting Ss. It is also possible that B may feel that his doubting during the early stages of the interaction was responsible for A's change in behavior. Thus, it was anticipated that B would maintain a relatively high rate of doubting during the last 32 trials, particularly in the $50 \%-0 \%$ condition. GAME

Each $S$ picks the top card from his own deck of shuffled cards. He observes the color of his card and then $B$ passes his card to A. After noting the color of the two cards, A informs $B$ whether the cards are the same or different in color. $B$ then has the option of either accepting or doubting A's message. If $B$ doubts the message, the cards are displayed and the outcomes are determined by whether the cards are in fact similar or different. In addition, $B$ knows whether A was lying or not. If B accepts the message, the trial is complete and the outcomes are determined by $A$ 's statement. In this case, the cards are not displayed. The outcome associated with each of the six events examined in the experimental game are presented in Fig. 1.

SUBJECTS AND PROCEDURE

The participants were 72 male and 64 female students enrolled in introductory psychology classes at the University of California, Los Angeles. Same-sex pairs were randomly assigned to one of the four treatment conditions.

When Ss entered the experimental room, they were seated in the two outside cubicles in a row of three, facing $E^{1}$ On the table in front of each $S$ were four decks of $504 \times 6$ in. index cards, on which were written, respectively, the words "doubt," "O.K.," "same," and "different." The $E$ handed each $S$ a deck of playing cards, told them that each deck contained 40 black and 40 red cards, told them to examine the cards, and then asked them to shuffle their cards.

The $E$ then indicated the slots in each partition to the Ss and thoroughly explained the game and the various outcomes to them. He told them that the values in the outcome table (Fig. 1) represented the number of points that could be earned on each trial. The Ss were told that $\mathrm{E}$ would sit in the middle cubicle, pass the playing 


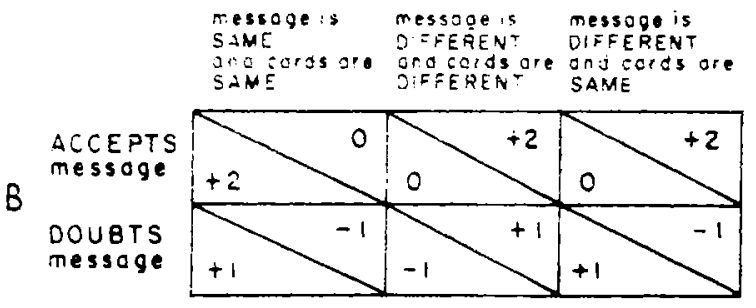

Fig. 1. Response consequences associated with the six outcomes examined in the experimental game.

cards, relay $S s^{\circ}$ messages, and record their messages and the color of the playing cards. Both $S$ s were trained in the $A$ and $B$ roles. This was done so that they might learn better the contingencies associated with the various moves and, in particular, might appreciate the decision dilemma faced by their opponent. After training was completed. E told the $S$ s that their goal during the interaction should be to try to maximize their own point total. The Ss then ostensibly drew for their roles during the game. Each $S$ picked a folded sheet of paper out of a bag. Unknown to them, both papers indicated that they would be Plaver $B$.

The $E$ then seated himself in the middle cubicle and, after the Ss had completed a brief pretask questionnaire, began. The $E$ played the role of Player A against both Ss. On each trial, after an appropriate pause, $E$ presented a card marked either "same" or "different" to both Ss and then recorded their responses " O.K." or "doubt"). If an $S$ returned a "doubt" message, E returned S's own card to him, together with an appropriately colored card from E's deck that completed the true color-matching state assigned to $\mathrm{S}$ for that trial. In each block of 16 trials, there were 8 true "same" and 8 true "different" states, randomly ordered. The E either did not lie at all $(0 \%)$ or lied two $(25 \%)$ or four $(50 \%)$ times in each block. For example, in the $25 \%$ condition, $E$ made eight honest "different" statements, six true "same" statements, and twice he said "different" when the cards were the same in color. The location in each block of the programmed false assertions was by random assignment. After the last (64th) trial, the Ss filled out a postinteraction questionnaire.

$$
\text { RESULTS }
$$

In addition to the lie sequence variable, the $S$ s were divided on the basis of their responses to the preinteraction question, "How trustworthy do you believe the other participant will be during the task?" Those $S s \quad(N=50)$ who rated the expected trus:worthiness of other as 1 ,
2 , or $3(1=$ extremely trustworthy and 7 = extremely untrustworthy) were treated as a separate population and labeled initially trusting; the remaining $86 \mathrm{Ss}, 52$ of whom indicated a 4 rating, were labeled initially suspicious. The focus of the following analysis is on the trials on which $A$ asserted that he should receive more points than $B$ (i.e., $A$ says "different"). The major dependent variable was the percentage of these trials on which B doubted A's assertion and was labeled doubting rate $(100 \times$ number of doubts/number of statements of "different" by A).

A Lie Sequence by Trust by Sex by Trial Block analysis of variance (least-squares solution, Winer, 1962) reveals that the doubting rate of the suspicious $\mathrm{Ss}$ was higher (48.4 vs 38.0 ) various lie sequences examined. than that of the trusting ones $(F=$ $3.94, \mathrm{df}=1 / 120, \mathrm{p}<.05)$. The same analysis also indicates a significant main effect of lie sequence $(F=11.5$, $\mathrm{df}=3 / 120, \mathrm{p}<.01)$. The 50\%-0\% sequence elicited a higher overall rate of doubting (56.5) than the other three patterns $(25.8,39.6,33.2$ to $0 \%-25 \%, \quad 0 \%-50 \%$, and $25 \%-0 \%$, respectively). The differences among the latter three patterns were not significant. However, individual comparisons indicate that the rate of doubting was significantly less when $A$ was initially honest $(0 \%-25 \%+$ $0 \%-50 \%$ ) as compared to when he was not $(25 \% \cdot 0 \%+50 \% .0 \%)$ and when his overall lie rate was $12 \%(0 \% .25 \%+$ $25 \%-0 \%)$ rather than $25 \%(0 \%-50 \%+$ $50 \%-0 \%$.

The Lie Sequence by Block interaction also was significant $(F=$ $7.94, \mathrm{df}=9 / 360, \quad \mathrm{p}<.01)$. The doubting rate of the Ss over blocks (of 16 trials) as a function of the four lie sequences examined in the present study is presented in Fig. 2. [Also shown for comparison are the rates of doubting by Ss to $0 \%, 25 \%$, and $50 \%$ constant rates of deception. The values shown for these treatment conditions are based on the results of the author's previously cited study (Benton et al, 1969). The $S$ population, instructions, and response consequence table used in that study were the same as in the present study.] Exposure to an honest

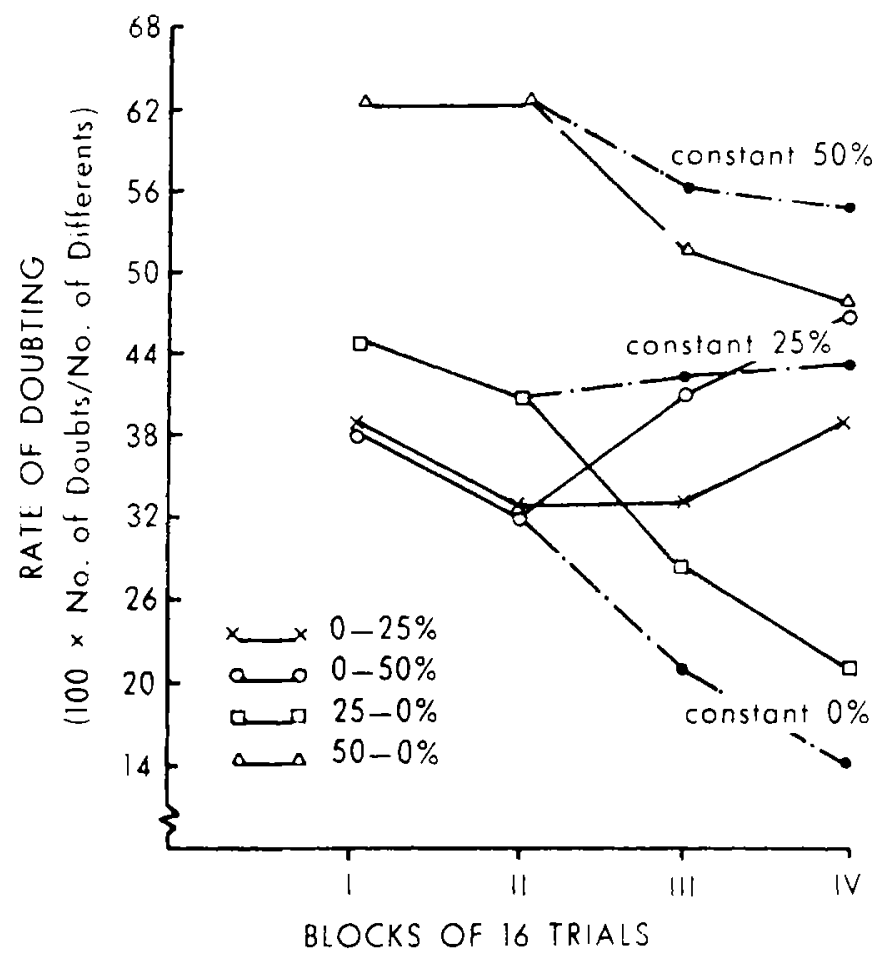

Fig. 2. Rate of doubting by B over blocks of 16 trials as a function of the 
$A$ resulted in a decrease in doubting from the first to the second block $(\mathrm{p}<.05)$, and the introduction subsequently of occasional lies $(25 \%)$ did not produce a sudden increase in doubting. Not until the last 16 trials did $S s$ in the $0 \%-25 \%$ condition significantly increase their rate of doubting, and then only to the level of the first 16 trials. The $S s$ in the $0 \%-50 \%$ condition, on the other hand, increased their rates of doubting during the third block of trials $(p<.01)$ and then again during the fourth block $(p<.05)$. It may be observed, however, that the Ss exposed to a constant $50 \%$ rate of lying manifested a higher rate of doubting throughout the 64 trials than that evidenced at any time by Ss in the $0 \%-50 \%$ condition. Perhaps if the interaction had been extended in the present study the $0 \%-50 \%$ Ss would have leveled off at the constant $50 \%$ rate.

The Ss in both the 25\%-0\% and the $50 \%-0 \%$ conditions maintained their doubt rates during the first 32 trials and then decreased them significantly during the third block $(p<.01)$ and again during the fourth block $(\mathrm{p}<.05)$. In addition, the $25 \%$ lie rate elicited a lower doubt rate during the first two blocks than did the $50 \%$ lie rate $(p<.01)$, and this difference continued durin' the last two blocks. The more the initial lying, the higher the initial and subsequent rate of doubting by the Ss

Analysis of the data from the point of view of the score achieved by $A$ as a function of the four experimental conditions indicates that $\mathrm{A}$ was least successful when he utilized the $50 \%-0 \%$ strategy $(50$ points $)$. The other three sequences were equally effective, and they produced the same number of points (56) as that previously reported (Benton et al, 1969 ) for the no-lie (0\%) strategy. There is, however, some indication (Sequence by Trust by Sex, $F=2.24$, df $=3 / 120, \mathrm{p}<.10)$ that the $0 \%-50 \%$ sequence is particularly effective $(64$ points) against females who expect $A$ to be trustworthy.

Finally, analyses of the Ss' postinteraction questionnaire ratings reveal a number of significant effects of overall lie rate with respect to the Ss' semantic differential ratings of $A$. An A who lied $12 \frac{1}{2} \%$ of the time $(0 \%-25 \%+25 \%-0 \%)$ was seen as less aggressive and more cooperative, honest, and active than one who lied $25 \%$ of the time, and he also was evaluated more highly (good-bad; positive-negative). A significant effect of early vs late lying with respect to the Ss' responses to the questions, "How successful do you feel you were in changing other's behavior?" and
"How responsible do you feel you were for the outcome of the interaction?" also was obtained. Ss felt more successful and responsible when A was honest during the last 32 trials $(25 \%-0 \%+50 \%-0 \%)$ rather than during the first 32 trials $(0 \%-25 \%+0 \%-50 \%)$.

\section{DISCUSSION}

There is no support in the results of this study for the hypothesis that information-dependent persons overreact when their source begins to lie after having behaved in a totally honest manner. Rather, the data suggest that they slowly adopt a rate of surveillance similar to that of persons exposed to a constant rate of the existing level of dishonesty. Of course, these conclusions must be qualified in terms of the particular pay off matrix values, incentive level, and lie patterns studied in the present investigation. For example, it is possible that $\mathrm{A}$ was not truthful for a long enough period of time for the rates of dishonesty sampled after Trial 32 to substantially violate the Ss' expectations. If this were the case, the competitive behavior of the other would not have been very frustrating or startling to the players. The main effect in this study of early honesty by A appears to have been to $F^{\prime \prime l l o n g ~ t h e ~}$ lowered doubt rate of the $S$ s in the $0 \%-25 \%$ condition. This finding is consistent with Pruitt's (1969) contention that decision makers are often hesitant to discard their previous interpretation of the motives of another party, even when faced with disconfirming evidence. The present author believes that the doubting rate results obtained for the $0 \%-25 \%$ and $0 \%-50 \%$ conditions primarily reflect the delay on the part of the $S s$ in discovering that $A$ had begun to lie. This seems likely, considering the already low doubt rate of the Ss and the relatively low number of lies made by $A$ during Trials 33-64. In the $0 \%-25 \%$ condition, only 2 of 10 "different" statements were lies and 7 of the $33 \mathrm{Ss}$ in this condition never "caught" A. Examination of the doubt rates of only those Ss who "caught" A at least once during the last 32 trials indicates that these Ss quickly adopted and maintained a similar rate of doubting during the third and fourth blocks of trials $(44 \%$ and $45 \%$, respectively) as that manifested by Ss exposed to a constant $25 \%$ rate of lying (Benton et al, 1969).

The results clearly indicate that the more A lied initially, the higher was the Ss' subsequent rate of doubting. It seems reasonable that detection of deceitful behavior should increase the Ss' suspicion of A's future statements. In addition, early detection may emphasize the necessity for continual monitoring of A's communications.
Also, a majority of the $25 \%-0 \%$ Ss and all of the $50 \%-0 \%$ Ss experienced partial reinforcement of their doubting during Blocks 1 and 2. This may have lowered the rate of extinction for doubting behavior.

The "delayed effect" (Oskamp, 1971) found in the present investigation is somewhat different from that obtained in prisoner's dilemma game studies. The results of those studies indicate that a change from a $0 \%$ cooperative strategy to a $100 \%$ cooperative strategy produces greater postchange cooperation than does a consistent $100 \%$ cooperative strategy (Oskamp, 1971). No such high level of initial competition or abrupt change in strategy was examined in the present study, and it is conceivable that a $100 \%-0 \%$ lie sequence would produce less postchange surveillance than would the 50\%-0\% sequence. Such a determination, however, requires further investigation.

Perhaps the most interesting of the posttask questionnaire data concerns Ss' responses with respect to their ability to change the other player and their feelings of responsibility for the game outcome. The Ss in the 50\%-0\% and $25 \%-0 \%$ conditions as compared to those in the $0 \%-50 \%$ and $0 \%-25 \%$ conditions reported that they were more successful and responsible. These results suggest that actors are more likely to attribute control to themselves when their behavior results in a positive state (honesty) than when the consequences are negative.

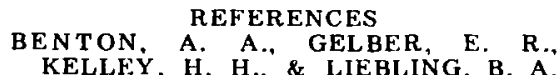
KELLEY, H. H., \& LIEBIING, B. A. Reactions to various degrees of deceit in a
mixed-motive relationship. Journal of Personality \& Social Psychology, 1969, $12,170-180$.

GRUDER, C. L. Relationships with opponent and partner in mixed-motive bargaining. Journal of Conflict Resolution, $1971,15.403-416$.

LINDSKOLD, S. BONOMA, T. V.. SCHLENKER, B. R.. \& TEDESCHI, J. T. Factors affecting the effectiveness of reward power. Psychonomic Science. $1972,26,68-70$.

LOOM IS, J. L. Communication, the development of trust, and cooperative behavior. Human Relations, 1959, 12 , 305-315.

OSKAMP S. Effects of programmed strategies on cooperation in the Prisoner's Dilemma and other mixed-motive games. Journal of Conflict Resolution, 1971, 15, 225-259.

PRUITT, D. G. Stability and sudden change in interpersonal and international affairs. Journal of Conflict Resolution, 1969,13 . 18-38.

TEDESCHL, J. T., BONOMA, T. V., \& BROWN, R. C. A paradigm for the study of coercive power. Journal of Conflict Resolution, 1971, 15, 197-223.

TEGER. A. I. The effects of early conperation on the escalation of conflict. Journal of Experimental Social 
Psychology. 1970, 6. 187-204.

WIXER, B. J. statistical principles in

trperimental design New York
McGraw-Hill. 1962.

NOTE

1. Eric R. Gelber and Barry A. Liebling served as Es, and their skillful handling of the role and assistance in organizing the data are gratefully acknowledged. 